\title{
Low calf circumference is associated with frailty in diabetic adults aged over 80 years
}

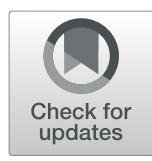

\author{
Yun-Xia Zhu ${ }^{\dagger}$, Yue Zhang ${ }^{\dagger}$, Yan-Yan Wang, Chen-Xi Ren, Jun Xu and Xiao-Yan Zhang ${ }^{*}$ (])
}

\begin{abstract}
Background: Frailty is now seen as a significant factor in older people with diabetes, whose mortality and disability increased. This study aims to investigate the association between calf circumference (CC) with frailty in diabetic adults aged over 80 years.

Methods: A cross-sectional analysis was performed on the data of 426 diabetic adults aged over 80 years. On admission, demographic data and laboratory parameters were recorded. CC was measured on the lower right leg at the point of the maximal circumference. All participants accepted frailty assessments. Frailty was mainly defined using the Fried frailty phenotype criteria.
\end{abstract}

Results: The CC levels were significantly lower in the frail than the non-frail $(26.7 \pm 4.0 \mathrm{vs.} 31.2 \pm 4.0, P<0.001)$. CC was negatively correlated with the Fried frailty phenotype index $(P<0.001)$. Logistic regression analysis of frailty revealed that age (Odds Ratio (OR), 1.368; 95\% Confidential Interval (Cl) 1.002-1.869; $P=0.049)$, CC (OR, 0.756; 95\%Cl 0.598-0.956; $P=0.019$ ) were independent impact factors of frailty after adjusting all the potential confounders. Participants with low CC tertile had a significantly higher Fried frailty phenotype index than those with high CC tertiles. The best CC cut-off value for predicting frailty was $29.3 \mathrm{~cm}$, its sensitivity was $75.0 \%$, and the specificity was $78.6 \%$, and areas under the curve (AUC) was $0.786(P<0.001)$.

Conclusions: CC was strongly related to frailty in diabetic adults aged over 80 years, suggesting that CC may be helpful for monitoring physical frailty in older adults in clinical and research settings.

Keywords: Calf circumference, Frailty, Aging, Diabetes

\section{Background}

Increasing diabetes and aging has become a global social, health, and economic burden, resulting in functional decline and physical disability of old adults. Diabetic patients already have an accelerated aging process that puts them at a higher risk for becoming frailty or frailtyrelated phenotype at an earlier age $[1,2]$. Frailty is a state of increased vulnerability to inner and external stressors and a limited capacity to recover [3]. A study from John Hopkins Medical Institutions involving 5317 participants aged over 65 reported that frailty is present

\footnotetext{
* Correspondence: zhangxy971088@hotmail.com

†Yun-Xia Zhu and Yue Zhang contributed equally to this work.

Department of Geriatrics, Shanghai Jiaotong University Affiliated Sixth

People's Hospital, Road Yi Shan 600, Shanghai 200233, China
}

in $25 \%$ of patients with diabetes, compared with a prevalence of $6.9 \%$ in the whole community-dwelling population [4]. Frailty is emerging as a new complication of diabetes in addition to the traditional microvascular and macrovascular complications leading to considerable disability [5]. Even frail diabetic patients had high mortality than their non-frail counterparts $[6,7]$.

Due to the focus on the function being more relevant for older patients, a key priority in managing older patients with diabetes is to delay or avoid the appearance of frailty [8]. Frailty is bidirectional, and with appropriate interventions, it can be reversed $[9,10]$. It appears to be a dynamic process with several intermediate stages that can improve or worsen over time, highlighting the need to detect it earlier to reduce potential health outcomes.

(c) The Author(s). 2020 Open Access This article is licensed under a Creative Commons Attribution 4.0 International License, which permits use, sharing, adaptation, distribution and reproduction in any medium or format, as long as you give appropriate credit to the original author(s) and the source, provide a link to the Creative Commons licence, and indicate if changes were made. The images or other third party material in this article are included in the article's Creative Commons licence, unless indicated otherwise in a credit line to the material. If material is not included in the article's Creative Commons licence and your intended use is not permitted by statutory regulation or exceeds the permitted use, you will need to obtain permission directly from the copyright holder. To view a copy of this licence, visit http://creativecommons.org/licenses/by/4.0/ The Creative Commons Public Domain Dedication waiver (http://creativecommons.org/publicdomain/zero/1.0/) applies to the data made available in this article, unless otherwise stated in a credit line to the data. 
Another question for why detection of frailty in diabetic patents is so essential is these patients whose risk of hypoglycemia increased, suggesting that reducing or even withdrawing hypoglycemic agents may be needed. Thus, early recognition of frailty and early intervention in older adults with diabetes should be critical in their primary care.

A few components of the frailty phenotype, including weight loss, weakness, slowness, reduced physical activity, and exhaustion, are direct symptoms of decreased muscle strength and function. Although the mechanism linking diabetes and frailty are poorly understood, the main factor explaining this link is sarcopenia, an agerelated loss of skeletal muscle mass, strength, and function. After 30 years old, human muscle mass decreases at an annual rate of $1-2 \%$. This tendency is accelerated to an annual rate of $1.5-3 \%$ after 60 years and even more rapidly over 75 years old [11]. The progressively decreased muscle mass and increased fat mass are various body compositional changes associated with aging [12]. A study showed skeletal muscle mass $(\mathrm{OR}=0.159$, $95 \%$ CI $0.064-0.396$ ) was a protective factor for frailty in 656 elder inpatients aged $\geq 65$ [13]. Calf circumference (CC) can reflect the skeletal muscle mass and is related to humans' physical function. It has been proven to be a simple marker for sarcopenia and nutritional risk in a few studies [14-16]. CC was negatively related to mortality risk and frailty index in the senior population [17]. A survey from México reported that low CC level is associated with a higher risk of death in frail patients [18].

Type 2 diabetes is known to cause accelerated muscle loss, predominantly in the lower limbs [19]. Insulin resistance, the primary mechanism of type 2 diabetes, has been recognized as a significant risk factor for frailty due to increased chronic inflammatory, impaired endothelial functioning, altered lipid metabolism, and atherosclerosis [20]. A study included 11,527 subjects aged 40-85 years from the National Health and Nutrition Examination Survey (NHANES) showed a significant negative correlation between the $\mathrm{CC}$ and homeostatic model assessment of insulin resistance (HOMA-IR) [21]. We hypothesis CC could be a potential indicator of diabetic frailty. As far as we know, there is still a lack of study to investigate CC's performance in predicting the frailty of diabetes until now.

This study tried to determine the association between $\mathrm{CC}$ and frailty in older diabetic adults over 80 years. People more than 80 years are overgrowing in many countries nowadays. These people are not routinely included in clinical studies, predominantly random control trials, due to their comorbidities, polypharmacy, or generally poor prognosis. However, in the real world, they are mainly frail diabetic patients. We primarily concern with the relationship between $\mathrm{CC}$ and frailty in this population.

\section{Methods Study population}

We screened 1226 consecutive, aged $\geq 80$ years patients admitted to our division between April 2017 through September 2018. The patients who were non-diabetes $(n=644)$, acute diabetes complication $(n=12)$, carcinomatous cachexia $(n=37)$, critical illness (including acute myocardial infarction, acute stroke, severe infection, etc) $(n=47)$, inability to communicate $(n=13)$, bedridden status $(n=35)$, and edema of lower extremity $(n=12)$ were further excluded (Supplemental Figure 1). In this cohort, we had studied that low CC could increase nutritional risk [16], and the malnutrition was an independent risk factor for mortality [22]. In addition, the Nutritional Risk Screening 2002 (NRS2002) score can independently predict the mortality compared with the Mini Nutritional Assessment Short Form (MNA-SF) over more than 2 years of follow-up [23]. In this study, we focused on the CC's performance in frailty in this cohort.

\section{Data collection}

A questionnaire is used to collect information on participants' demographic data, chronic disease history, smoking, and alcohol drinking history (Supplemental Table 1). Examining medical records were further used to verify the data.

\section{Frailty diagnosis criteria}

Frailty was mainly defined using the Fried frailty phenotype [4] and detailed according to "Chinese expert consensus on assessment and intervention for elderly patients with frailty" (Supplemental Figure 2). The total frailty index (range: $0-5$ ) was calculated by allocating 1 to positive responses on each of the five components (Weight loss, Slowness, Weakness, Low activity, and Exhaustion). Participants with a score of $\geq 3$ were diagnosed as frailty.

\section{Anthropometric measurement}

Height, body weight, and waist circumference (WC) were measured by standard methods [24]. Body Mass Index (BMI) was calculated as weight divided by height squared $\left(\mathrm{kg} / \mathrm{m}^{2}\right)$. The lower right leg's greatest circumference was measured in patients' standing position as CC. For each examination, we measured three times to obtain the mean value for further analysis.

\section{Handgrip strength}

The handgrip strength of the dominant hand was the maximum value measured three times with WCS-100 
electronic vibrometer. At least $1 \mathrm{~min}$ rest was required between each test.

\section{Laboratory measurements}

All the participants took the collection of blood samples after overnight fasting. Serum Albumin, Creatinine, Hemoglobin, glycosylated hemoglobin A1c (HbA1c) levels were tested, as described in our previous publication [16].

\section{Statistical analyses}

The continuous variables following normal distribution were expressed with mean \pm standard deviation. The Student's t-test was used to measure the difference between groups (BMI, Waist circumference, Calf circumference, Albumin, Hemoglobin, and HbA1c). For non-normal distributed variables, the median / lowerupper quartile range was used. The Mann-Whitney $U$ test was conducted to measure the intergroup difference (Handgrip strength and Creatinine). Categorical variables were showed with frequency percentage, and the chi-square test was conducted for intergroup comparison (Sex). We further applied Pearson (Age, BMI, Waist circumference, Albumin, Hemoglobin, HbA1c, and Fried phenotype frailty Index) or Spearman (Creatinine and Handgrip strength) correlation analysis to analyze the association between CC and other parameters. Multiple logistic regression analysis was performed to determine the risk factors of frailty. We categorized the patients into tertile and further applied the ANOVA test over the Fried frailty phenotype index. The receiver operating characteristic (ROC) curve was used to identify the optimal CC cut-off points to predict frailty. SPSS 21.0 software was used to do all the data analyses.

\section{Results}

\section{General characteristics of the participants}

We enrolled 426 patients (mean age, $86.7 \pm 4.3$ years; male/female, 305/121) in this study. More than half of the patients were frailty $(57.75 \%, n=246)$. Table 1 showed the frail and non-frail subjects' clinical characteristics. The age, Handgrip strength, Albumin, Hemoglobin, HbA1c, and Creatinine were significantly different between the two groups $(P=0.048,0.012,0.003,0.014,0.025$, and 0.032 , respectively). However, the sex, $\mathrm{WC}$, and BMI were not significantly different between the frail and non-frail $(P=0.174,0.296$, and 0.323 , respectively).

\section{The difference of CC between frail and non-frail participants}

When using Fried frailty phenotype diagnosis criteria, 246 cases were divided as frail. CC levels were significantly different between frail and non-frail group $(26.7 \pm$ 4.0 vs. $31.2 \pm 4.0, P<0.001$ ) (Fig. 1 ).

\section{Factors associated with CC}

It was showed in Table 2 that CC was significantly negatively correlated with age and the Fried frailty phenotype index $(P=0.041$, and $<0.001$, respectively $)$. However, CC was significantly positively related to BMI, WC, Hemoglobin, Albumin, HbA1c, and Handgrip strength $(P=0.004,<0.001,<0.001,<0.001,<0.001$, and 0.013 , respectively).

\section{Factors associated with frailty}

We calculated the OR (95\% CI) of CC associated with frailty in four logistic regression models (Table 3): model 1: adjusted for age and sex; model 2: adjusted for anthropometric indicators (BMI, WC, and HGS); model 3: adjusted for biological markers (Hemoglobin, Albumin, Creatinine, and HbA1c); model 4: adjusted for all the above potential confounders. CC was related to frailty in each adjusted model. In model 4 , after adjusting all the confounders, it was revealed that age (OR, 1.368; 95\%CI $1.002-1.869$; $P=0.049)$, CC (OR, 0.756; 95\%CI $0.598-$ $0.956 ; P=0.019)$ were independent risk factors of frailty.

Table 1 Clinical and biochemical characteristics of frail and non-frail old diabetic adults

\begin{tabular}{llll}
\hline variable & $\begin{array}{l}\text { frail } \\
(\boldsymbol{n}=\mathbf{2 4 6})\end{array}$ & $\begin{array}{l}\text { non-frail } \\
(\boldsymbol{n}=\mathbf{1 8 0})\end{array}$ & $\boldsymbol{P}$ \\
\hline Age (years) & $88.3 \pm 3.4$ & $86.4 \pm 3.8$ & 73.53 \\
Sex (male\%) & 68.89 & $24.03 \pm 3.39$ & 0.048 \\
Body mass index $\left(\mathrm{kg} / \mathrm{m}^{2}\right)$ & $22.89 \pm 3.45$ & $91.96 \pm 10.67$ & 0.174 \\
Waist circumference $(\mathrm{cm})$ & $89.05 \pm 11.82$ & $20.12(14.66-23.16)$ & 0.323 \\
Handgrip strength $(\mathrm{kg})$ & $14.43(6.26-17.53)$ & $41.00 \pm 4.22$ & 0.012 \\
Albumin(g/dl) & $37.09 \pm 6.44$ & $119.86 \pm 17.53$ & 0.003 \\
Hemoglobin(g/dL) & $107.51 \pm 21.49$ & $7.12 \pm 1.11$ & 0.014 \\
HbA1c(\%) & $6.48 \pm 0.99$ & $81.00(64.00-104.00)$ & 0.025 \\
Creatinine $(\mu \mathrm{mol} / \mathrm{L})$ & $93.00(76.50-113.00)$ & 0.032 \\
\hline
\end{tabular}




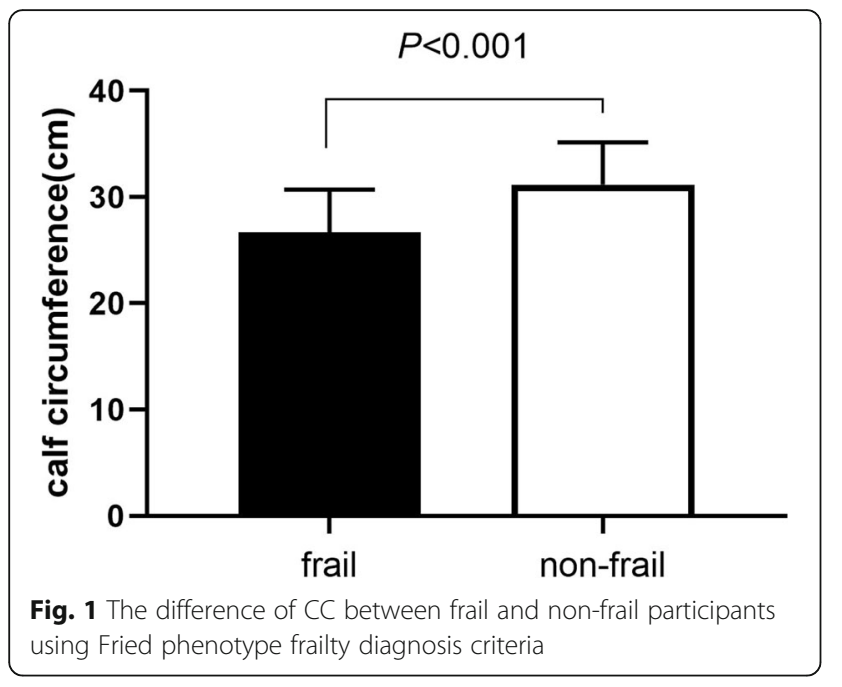

The difference of Fried frailty phenotype index according to $\mathrm{CC}$ tertile

We compared The Fried frailty phenotype index according to $\mathrm{CC}$ tertile. Compared with patients in tertile $3(\geq 30.1 \mathrm{~cm})$, the Fried frailty phenotype index was significantly higher among participants in tertile $2(26.0-30.1 \mathrm{~cm})$ and tertile $1(<26.0 \mathrm{~cm})$ ( $\mathrm{P}$ for trend $<0.001$ ) (Fig. 2).

\section{The cut-off value of $\mathrm{CC}$ for frailty}

ROC curve analysis was used to find CC's best cut-off value for identifying frailty in older diabetic patients. The best CC cut-off value was $29.3 \mathrm{~cm}$, the AUC was 0.786 (95\% CI: 0.669-0.904), the Youden index was 0.536 , with a $75 \%$ sensitivity, and $78.6 \%$ specificity. $(P<$ 0.001) (Fig. 3).

\section{Discussion}

In this study, we provided the evidence for the first time that decreased CC levels were associated with frailty in type 2 diabetic patients aged over 80 years independent

Table 2 Correlation analysis of clinical and biochemical parameters with calf circumference

\begin{tabular}{lll}
\hline variable & $\boldsymbol{r}$ & $\boldsymbol{P}$ \\
\hline Age & -0.252 & 0.041 \\
Body mass index & 0.469 & 0.004 \\
Waist circumference & 0.540 & $<0.001$ \\
Handgrip strength & 0.688 & $<0.001$ \\
Albumin & 0.531 & $<0.001$ \\
Hemoglobin & 0.484 & $<0.001$ \\
Creatinine & 0.143 & 0.256 \\
HbA1c & 0.371 & 0.013 \\
Fried phenotype frailty Index & -0.584 & $<0.001$ \\
\hline
\end{tabular}

of established risk factors. CC levels significantly reduced in type 2 diabetes with frail than their counterparts. CC was negatively correlated with the Fried frailty phenotype index. CC was an independent risk factor of frailty, and the Fried frailty phenotype index increased with a reduction in CC levels. The optimal CC cut-off point for predicting frailty in type 2 diabetic adults aged over 80 years was $29.3 \mathrm{~cm}$ in this population.

Frailty is an emerging complication in older people with diabetes [25]. The emergence of frailty may alter the natural history of diabetes from a progressive to a regressive trajectory. Frailty has a triple meaning in older adults with diabetes: a symptom, a prognostic marker, and a therapeutic goal. Our study showed a significant difference in age, Handgrip strength, Albumin, and Hemoglobin between the frail diabetic patients and their counterparts, suggesting that reverse metabolism due to malnutrition in the elderly diabetic patients might be involved. In this study, the old frail type 2 diabetes had a lower HbA1c. There is a consensus that intensive glucose lowering has limited benefits and may be harmful to diabetic older people [26]. It was showed that functional performances over 2 years are better in frail individuals with an $\mathrm{HbA} 1 \mathrm{c}>8.0 \%$ than those with $\mathrm{HbA} 1 \mathrm{c}$ between $7.0-7.9 \%$ [27]. A Japanese study showed a relatively lower HbA1c level is a risk factor for frailty, independent of anemia [28]. However, a previous study showed a U-shaped curve of the HbAlc level was a frailty risk factor in patients with type 2 diabetes [29]. Prospective studies need to be done in a massive population to illustrate the relationship between HbA1c and frailty in the old diabetic population.

Anthropometric measurements are usually performed in clinical practice, just like BMI, widely accepted and used. There is scarce commonly used anthropometric measurements on the association with frailty. In this study, there was no difference between frail and nonfrail in BMI and WC levels. Usually, at the diabetes early stage, many patients are overweight or obese. However, as people aging, their appetite, caloric need, and energy consumption reduced. They begin to lose weight and become less active, and the potential for frailty occurs. As older adults lose weight and frailty develops, there is an increase in insulin sensitivity, and glucose tolerance improves as visceral fat lost. Indexes of fat distribution such as BMI and WC might be related to adverse health outcomes, mainly due to excessive adiposity in the general population [30]. On the contrary, CC is much more directly associated with a lack of muscle mass than the other anthropometric index. So anthropometric measurements may have different significance in frail old adults.

Muscles play various essential roles in the human body; thus, loss of muscle mass and strength can cause a 
Table 3 Independent factors for frailty by multivariable logistic regression analysis

\begin{tabular}{|c|c|c|c|c|c|c|c|c|}
\hline \multirow[t]{2}{*}{ variable } & \multicolumn{2}{|l|}{ Model 1} & \multicolumn{2}{|l|}{ Model 2} & \multicolumn{2}{|l|}{ Mode 3} & \multicolumn{2}{|l|}{ Model 4} \\
\hline & $\mathrm{OR}(95 \% \mathrm{Cl})$ & $P$ & $\mathrm{OR}(95 \% \mathrm{Cl})$ & $P$ & $\mathrm{OR}(95 \% \mathrm{Cl})$ & $P$ & $\mathrm{OR}(95 \% \mathrm{Cl})$ & $P$ \\
\hline Age & $1.438(1.075-1.923)$ & 0.014 & & & & & $1.368(1.002-1.869)$ & 0.049 \\
\hline Sex & $0.659(0.053-8.347)$ & 0.747 & & & & & $0.780(0.432-1.409)$ & 0.410 \\
\hline BMI & & & $1.180(0.903-1.542)$ & 0.225 & & & $0.945(0.789-1.132)$ & 0.538 \\
\hline WC & & & $0.876(0.713-1.076)$ & 0.207 & & & 0.977 (0.934-1.023) & 0.320 \\
\hline HGS & & & $0.965(0.898-1.037)$ & 0.335 & & & $0.981(0.963-1.000)$ & 0.051 \\
\hline CC & $0.703(0.533-0.928)$ & 0.013 & $0.718(0.542-0.951)$ & 0.021 & $0.714(0.522-0.977)$ & 0.035 & $0.756(0.598-0.956)$ & 0.019 \\
\hline Alb & & & & & $0.748(0.476-1.176)$ & 0.209 & $0.890(0.735-1.079)$ & 0.236 \\
\hline $\mathrm{Hb}$ & & & & & $1.008(0.932-1.090)$ & 0.839 & $0.996(0.952-1.041)$ & 0.834 \\
\hline $\mathrm{HbA1c}$ & & & & & $0.550(0.278-1.369)$ & 0.120 & $0.695(0.233-2.071)$ & 0.514 \\
\hline $\mathrm{Cr}$ & & & & & $1.005(0.975-1.090)$ & 0.766 & $1.096(0.791-1.520)$ & 0.514 \\
\hline
\end{tabular}

BMI Body mass index; WC Waist circumference; HGS Handgrip strength; CC Calf circumference; Alb Albumin; $\mathrm{Hb}$ Hemoglobin; $\mathrm{Cr}$ Creatinine

Model 1 was adjusted for age and sex;

Model 2 was adjusted for BMI, WC, and HGS;

Model 3 was adjusted for Alb, $\mathrm{Hb}, \mathrm{HbA1C}$, and $\mathrm{Cr}$;

Model 4 was adjusted for all the above confounders

diverse range of functional disability and metabolic derangements in older adults. The pathogenic linkage between diabetes and frailty potentially includes premature senescence of organ systems, chronic low-grade inflammation, advanced glycosylation end products (AGEs) accumulation, and insulin resistance [31]. Elevation of low-grade systemic inflammation leads to muscle protein breakdown. Diabetes is associated with an increase in inflammatory cytokines, such as tumor necrosis factor and interleukin 6 have detrimental effects on muscle mass, strength, and physical performance in older adults [32, 33]. As a potential diabetic frailty

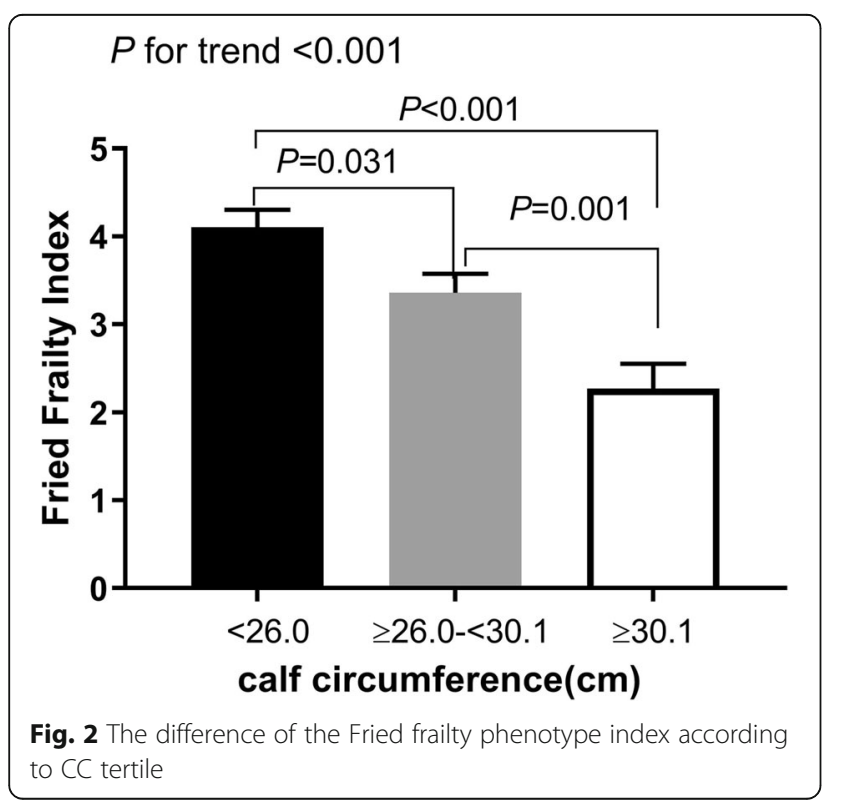

indicator, CC could be easily accessible to predict frailty, especially in communities and primary care settings.

Given the relevance of $\mathrm{CC}$ to an individual's robustness and well-being, identifying clear thresholds for CC and frailty is currently top research and clinical priority. However, anthropometric indices, including $\mathrm{CC}$, tend to vary by age, gender, ethnicity, and environment, making it challenging to determine common values. Asia Working Group for Sarcopenia 2019 (AWGS 2019) recommends that CC could be used to screen sarcopenia, and the cut-off value is $<34 \mathrm{~cm}$ for men and $<33 \mathrm{~cm}$ for women. While the European Working Group on Sarcopenia in Older People (EWGSOP2) recommends CC may be used as a surrogate for old adults when other muscle mass evaluation methods impossible, the cut-off is $<31 \mathrm{~cm}$ [34]. Different values of muscle mass and strength depending on ethnicity support the need for establishing a substantial region-specific cut-off value of CC. In this study, the best CC cut-off value for predicting frailty according to the Fried frailty phenotype was $29.3 \mathrm{~cm}$, with relatively high sensitivity and specificity. In our previous study, we found in aged $\geq 80$ years male, the optimal CC cut-off point for predicting nutritional risk was $29.75 \mathrm{~cm}$; for women was $28.25 \mathrm{~cm}$ [16]. Due to the low female percentage $(31.11 \%)$ in this study, we could not investigate the potential sex-specific effects. It seems the cut-off value of $\mathrm{CC}$ to predict diabetic frailty was higher than the cut-off value to predict nutritional risk. That means for diabetic patients, even at relatively higher CC, their frailty risk has increased.

There were some strengths to this study. Firstly, we focused on subjects aged over 80 years, who are routinely excluded in a lot of studies. On the contrary, they are 


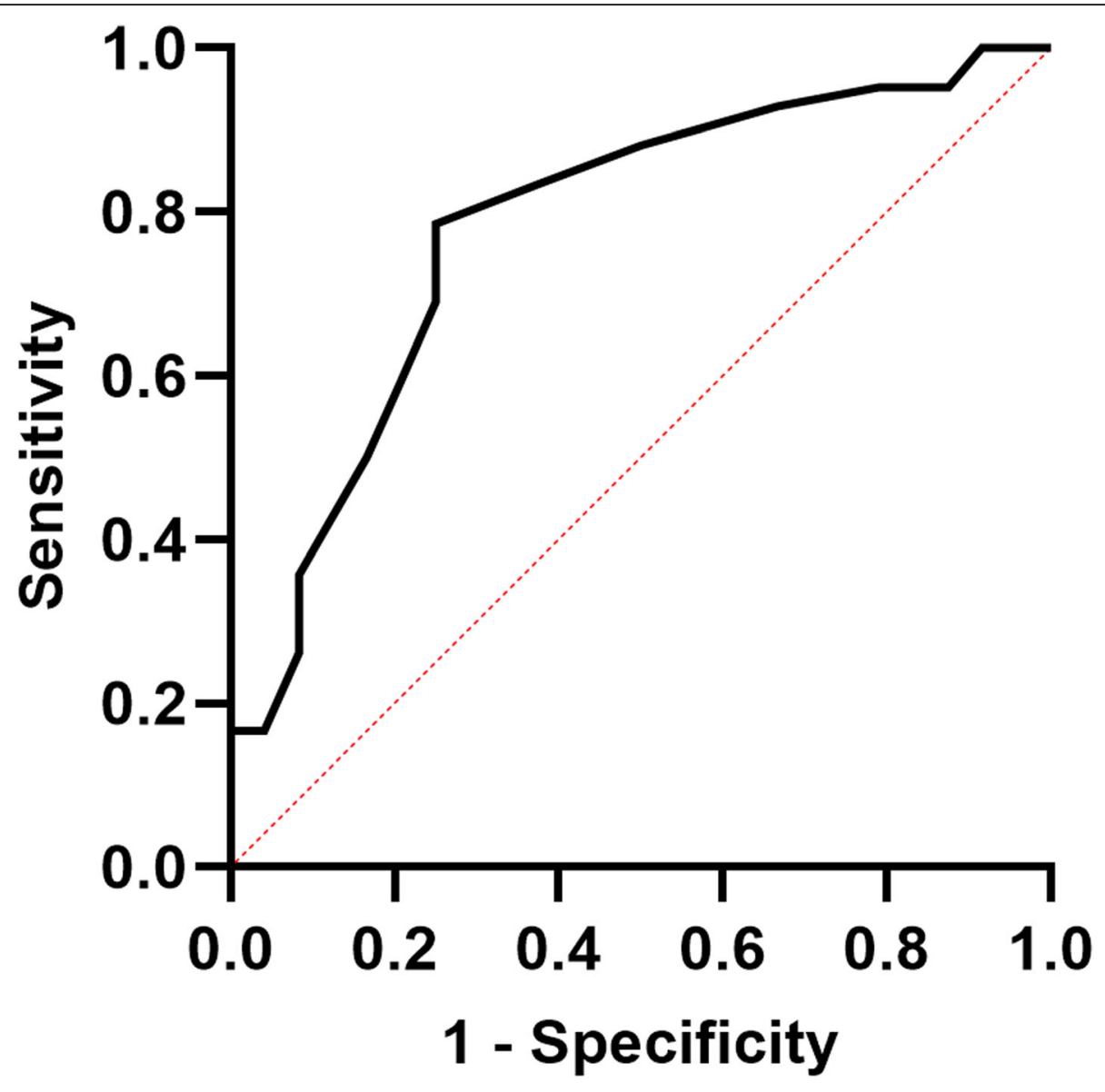

Fig. 3 ROC curve analysis of CC for frailty. AUC $=0.786(P<0.001)$; 95\% Cl, 0.669-0.904; CC cut-off point $=29.3 \mathrm{~cm}$; Youden index =0.536; sensitivity, $75.0 \%$; Specificity, $78.6 \%$

the mainly frail population in the real world. Secondly, some common potential risk factors of frailty had been adjusted, like Albumin, Hemoglobin, and Creatinine. Thirdly, it is a relatively large study of subjects with diabetic patients over 80 years old.

There are many limitations to our study. Firstly, as a cross-sectional and retrospective study, it could not evaluate any cause-effect relationship between CC and frailty. Secondly, there may be sex-specific effects exist between $\mathrm{CC}$ and frailty, due to lower female subject ratio in our study, we could not find this. Thirdly, some potential influencing factors on frailty were not considered. For example, all the participants were senior people, some medical history maybe not accurate, like the duration of diabetes. Likewise, the medicine they took were not included in this study. Prospective studies are needed to confirm the association between $\mathrm{CC}$ and frailty.

\section{Conclusions}

It is essential to focus on frailty in elderly diabetes patients and intervene in time. As far as we know, this study firstly reported that low CC was strongly associated with frailty in old adults over 80 years, for whom CC may be used as a proxy of frailty. In clinical and research practice, CC may be helpful for monitoring physical frailty in the senior population.

\section{Supplementary information}

Supplementary information accompanies this paper at https://doi.org/10. 1186/s12877-020-01830-2.

Additional file 1 Supplemental Figure 1: Flowchart for subjects enrolled in this study. Supplemental Figure 2: Frailty diagnosis criteria. BMI: Body Mass Index. MLTA: Minnesota Leisure Time Physical Activity Questionnaire. Supplemental Table 1: Participants Assessment Form.

\section{Abbreviations}

Alb: Albumin; BMl: Body mass index; CC: Calf circumference; Cr: Creatinine; HbA1c: Glycosylated hemoglobin A1c; Hb: Hemoglobin; ROC: Receiver operating characteristic; WC: Waist circumference

\section{Acknowledgements}

We thank all the nurses in our division who contribute to data collection and communicate with the subjects. 


\section{Authors' contributions}

The manuscript was conceived by authors XZ and YZ1, YZZ, YW, CR, JX contributed to finish the project and acquisition of the data. $Y Z 1$ and $Y Z 2$ performed the statistical analysis. YZ1 drafted the manuscript, XZ, YZ2 and YW revised the manuscript. All the authors approved this version for publication.

\section{Funding}

Not applicable.

\section{Availability of data and materials}

Not applicable.

\section{Ethics approval and consent to participate}

The Ethics Committee of the Shanghai Jiaotong University Affiliated Sixth People's Hospital [2016-141-(1)] approved this study. Written informed consent was obtained from all participants.

\section{Consent for publication}

Not applicable.

\section{Competing interests}

All the authors declare no conflicts of interest.

Received: 28 July 2020 Accepted: 13 October 2020

Published online: 19 October 2020

\section{References}

1. Assar ME, Laosa O, Rodríguez Mañas L. Diabetes and frailty. Curr Opin Clin Nutr Metab Care. 2019;22(1):52-7.

2. Kotsani M, Chatziadamidou T, Economides D, Benetos A. Higher prevalence and earlier appearance of geriatric phenotypes in old adults with type 2 diabetes mellitus. Diabetes Res Clin Pract. 2018;135:206-17.

3. Bortz WM 2nd. A conceptual framework of frailty: a review. J Gerontol A Biol Sci Med Sci. 2002;57(5):M283-8.

4. Fried LP, Tangen CM, Walston J, Newman AB, Hirsch C, Gottdiener J, Seeman T, Tracy R, Kop WJ, Burke G, et al. Frailty in older adults: evidence for a phenotype. J Gerontol A Biol Sci Med Sci. 2001;56(3):M146-56.

5. Sinclair AJ, Abdelhafiz AH, Rodríguez-Mañas L. Frailty and sarcopenia newly emerging and high impact complications of diabetes. J Diabetes Complicat. 2017:31(9):1465-73.

6. Ulley J, Abdelhafiz AH. Frailty predicts adverse outcomes in older people with diabetes. Practitioner. 2017:261(1800):17-20.

7. Chao CT, Wang J, Chien KL. Both pre-frailty and frailty increase healthcare utilization and adverse health outcomes in patients with type 2 diabetes mellitus. Cardiovasc Diabetol. 2018;17(1):130.

8. Aguayo GA, Hulman A, Vaillant MT, Donneau AF, Schritz A, Stranges S, Malisoux L, Huiart L, Guillaume M, Sabia S, et al. Prospective association among diabetes diagnosis, $\mathrm{HbA}$ (1c), Glycemia, and frailty trajectories in an elderly population. Diabetes Care. 2019;42(10):1903-11.

9. Bray NW, Smart RR, Jakobi JM, Jones GR. Exercise prescription to reverse frailty. Appl Physiol Nutr Metab. 2016:41(10):1112-6.

10. Clegg A, Young J, lliffe S, Rikkert MO, Rockwood K. Frailty in elderly people Lancet. 2013;381(9868):752-62

11. Hughes VA, Frontera WR, Roubenoff R, Evans WJ, Singh MA. Longitudinal changes in body composition in older men and women: role of body weight change and physical activity. Am J Clin Nutr. 2002;76(2):473-81.

12. Newman AB, Lee JS, Visser M, Goodpaster BH, Kritchevsky SB, Tylavsky FA, Nevitt M, Harris TB. Weight change and the conservation of lean mass in old age: the health, aging and body composition study. Am J Clin Nutr. 2005:82(4):872-8 quiz 915-876.

13. Xu L, Zhang J, Shen S, Hong $X$, Zeng $X$, Yang Y, Liu Z, Chen L, Chen X. Association between body composition and frailty in elder inpatients. Clin Interv Aging. 2020;15:313-20.

14. Maeda K, Koga T, Nasu T, Takaki M, Akagi J. Predictive accuracy of calf circumference measurements to detect decreased skeletal muscle mass and European Society for Clinical Nutrition and Metabolism-Defined Malnutrition in hospitalized older patients. Ann Nutr Metab. 2017;71(1-2):10-5.

15. Kawakami R, Murakami H, Sanada K, Tanaka N, Sawada SS, Tabata I, Higuchi M, Miyachi M. Calf circumference as a surrogate marker of muscle mass for diagnosing sarcopenia in Japanese men and women. Geriatr Gerontol Int. 2015:15(8):969-76.

16. Zhang XY, Zhang XL, Zhu YX, Tao J, Zhang Z, Zhang Y, Wang YY, Ke YY, Ren $C X, X u$ J, et al. Low calf circumference predicts nutritional risks in hospitalized patients aged more than 80 years. Biomed Environ Sci. 2019;32(8):571-7.

17. Landi F, Onder G, Russo A, Liperoti R, Tosato M, Martone AM, Capoluongo E Bernabei R. Calf circumference, frailty and physical performance among older adults living in the community. Clin Nutr. 2014;33(3):539-44.

18. Easton JF, Stephens CR, Román-Sicilia H, Cesari M, Pérez-Zepeda MU. Anthropometric measurements and mortality in frail older adults. Exp Gerontol. 2018:110:61-6.

19. Park SW, Goodpaster BH, Lee JS, Kuller LH, Boudreau R, de Rekeneire N, Harris TB, Kritchevsky S, Tylavsky FA, Nevitt M, et al. Excessive loss of skeletal muscle mass in older adults with type 2 diabetes. Diabetes Care. 2009;32(11):1993-7.

20. Abbatecola AM, Paolisso G. Is there a relationship between insulin resistance and frailty syndrome? Curr Pharm Des. 2008;14(4):405-10.

21. Chao YP, Kao TW, Chang YW, Peng TC, Chen WL, Wu LW. Utilization of anthropometric parameters as a novel tool for detection of insulin resistance. Clin Nutr. 2020:39(8):2571-9.

22. Tao J, Ke YY, Zhang Z, Zhang Y, Wang YY, Ren CX, Xu J, Zhu YX, Zhang XL, Zhang $X Y$. Comparison of the value of malnutrition and sarcopenia for predicting mortality in hospitalized old adults over 80 years. Exp Gerontol. 2020;138:111007.

23. Zhang $X$, Zhang $X$, Zhu $Y$, Tao J, Zhang Z, Zhang $Y$, Wang $Y$, Ke $Y$, Ren $C$, Xu J. Predictive value of nutritional risk screening 2002 and mini nutritional assessment short form in mortality in Chinese hospitalized geriatric patients. Clin Interv Aging. 2020;15:441-9.

24. Pan XR, Yang WY, Li GW, Liu J: Prevalence of diabetes and its risk factors in China, 1994. National Diabetes Prevention and control cooperative group. Diabetes Care 1997, 20(11):1664-1669.

25. García-Esquinas E, Graciani A, Guallar-Castillón P, López-García E, RodríguezMañas L, Rodríguez-Artalejo F. Diabetes and risk of frailty and its potential mechanisms: a prospective cohort study of older adults. J Am Med Dir Assoc. 2015;16(9):748-54.

26. Boussageon $\mathrm{R}$, Bejan-Angoulvant $\mathrm{T}$, Saadatian-Elahi M, Lafont $\mathrm{S}$, Bergeonneau C, Kassaï B, Erpeldinger S, Wright JM, Gueyffier F, Cornu C. Effect of intensive glucose lowering treatment on all cause mortality, cardiovascular death, and microvascular events in type 2 diabetes: meta-analysis of randomised controlled trials. Bmj. 2011;343:d4169.

27. Yau CK, Eng C, Cenzer IS, Boscardin WJ, Rice-Trumble K, Lee SJ. Glycosylated hemoglobin and functional decline in community-dwelling nursing homeeligible elderly adults with diabetes mellitus. J Am Geriatr Soc. 2012;60(7): 1215-21.

28. Yanagita I, Fujihara Y, Eda T, Tajima M, Yonemura K, Kawajiri T, Yamaguchi $\mathrm{N}$, Asakawa H, Nei Y, Kayashima Y, et al. Low glycated hemoglobin level is associated with severity of frailty in Japanese elderly diabetes patients. $J$ Diabetes Investig. 2018:9(2):419-25.

29. Zaslavsky O, Walker RL, Crane PK, Gray SL, Larson EB. Glucose levels and risk of frailty. J Gerontol A Biol Sci Med Sci. 2016;71(9):1223-9.

30. Lee $\mathrm{JH}$, Jun HS. Role of Myokines in regulating skeletal muscle mass and function. Front Physiol. 2019;10:42

31. Casanova F, Adingupu DD, Adams F, Gooding KM, Looker HC, Aizawa K, Dove F, Elyas S, Belch JJF, Gates PE, et al. The impact of cardiovascular co-morbidities and duration of diabetes on the association between microvascular function and glycaemic control. Cardiovasc Diabetol. 2017;16(1):114.

32. Visser M, Pahor M, Taaffe DR, Goodpaster BH, Simonsick EM, Newman AB, Nevitt M, Harris TB. Relationship of interleukin- 6 and tumor necrosis factoralpha with muscle mass and muscle strength in elderly men and women: the health ABC study. J Gerontol A Biol Sci Med Sci. 2002;57(5):M326-32.

33. Cesari M, Penninx BW, Pahor M, Lauretani F, Corsi AM, Rhys Williams G, Guralnik $J M$, Ferrucci L. Inflammatory markers and physical performance in older persons: the InCHIANTI study. J Gerontol A Biol Sci Med Sci. 2004;59(3):242-8.

34. Cruz-Jentoft AJ, Bahat G, Bauer J, Boirie Y, Bruyère O, Cederholm T, Cooper C, Landi F, Rolland Y, Sayer AA, et al. Sarcopenia: revised European consensus on definition and diagnosis. Age Ageing. 2019;48(1):16-31.

\section{Publisher's Note}

Springer Nature remains neutral with regard to jurisdictional claims in published maps and institutional affiliations. 